\title{
Extended-duration thromboprophylaxis after ventral hernia repair: a risk model to predict venous thrombotic events after hospital discharge
}

\author{
S. B. Kumar' ${ }^{1}$. D. Mettupalli' ${ }^{2}$ J. T. Carter ${ }^{3}$
}

Received: 22 May 2021 / Accepted: 22 July 2021 / Published online: 15 August 2021

(c) The Author(s) 2021

\begin{abstract}
Background Venous thromboembolism (VTE) is a major source of morbidity and mortality after ventral hernia surgery, but the risk of VTE after discharge has not been reported.

Study design Data from the American College of Surgeons-National Surgical Quality Improvement Program (ACS-NSQIP) were used to investigate the risk of post-discharge VTE. Current procedural terminology (CPT) codes identified all reported patients who underwent ventral hernia repair from 2011 to 2017. We created a multivariable regression model for postdischarge VTE, using the 2011-2016 dataset to develop the model and 2017 as a validation set. The prediction model was used to create a risk calculator as a mobile application.

Results The rate of VTE after surgery was $0.62 \%$ (878 of 141,065) with $48 \%$ occurring after discharge from the hospital. The final predictor model consisted of eight variables: age $>60$ years, male sex, body mass index $(\mathrm{BMI}) \geq 35 \mathrm{~kg} / \mathrm{m}^{2}$ ), operative time $>2 \mathrm{~h}$, concurrent panniculectomy, post-operative hospitalization $>1$ day, presence of bleeding disorder, and emergency operation. The model had good calibration and discrimination (Hosmer-Lemeshow goodness-of-fit test, $p=0.71$; $c$-statistic $=0.71)$. Threshold analysis showed a strategy of extended-duration thromboprophylaxis was optimized when the risk of post-discharge VTE was $>0.3 \%$.

Conclusion Forty-eight percent of VTEs after ventral hernia repair occur after discharge, particularly in older, male, obese patients undergoing longer and complex operations that require hospitalization $>1$ day. Post-discharge thromboprophylaxis should be considered in these patients, particularly when risk of VTE exceeds $0.3 \%$.
\end{abstract}

Keywords Ventral hernia repair · Venous thromboembolism $\cdot$ Risk prediction $\cdot$ Thromboprophylaxis

\section{Introduction}

Venous thromboembolism (VTE), defined as a deep vein thrombosis, pulmonary embolus, or both, remains a major source of morbidity and mortality after abdominal surgery [1]. Hernia patients are no exception [2,3]. Because one-third of all VTEs occur after surgery and effective

S. B. Kumar

sandhya.kumar@ucsf.edu

1 Department of Surgery, University of California San Francisco-Zuckerberg San Francisco General Hospital, 1001 Potrero Avenue, Suite 3A, San Francisco, CA 94110, USA

2 University of California, Berkeley, Berkeley, CA, USA

3 Department of Surgery, University of California San Francisco, San Francisco, CA, USA risk-reduction strategies exist, thromboprophylaxis strategies should be considered after every surgical procedure. Current CHEST guidelines recommend either mechanical thromboprophylaxis (such as sequential compression devices), chemothromboprophylaxis (i.e., unfractionated heparin, low-molecular weight heparin, or other agents); or both-depending on the degree of thrombotic risk and the overall risk of bleeding. For most patients, including hernia patients, CHEST guidelines recommend these prophylactic strategies to continue only while the patient is hospitalized, under the assumption that once a patient can walk easily after discharge, their risk of VTE has returned to baseline [1].

In the past decade, however, this assumption has been challenged. Many VTEs following surgery occur after discharge from the hospital in patients who are presumably walking [4-7]. Some reports have shown increased risk of 
VTE up to 90 days after surgery $[3,5]$. For example, in patients undergoing bariatric surgery, one report found that $80 \%$ of VTEs occurred after discharge [5]. Cancer patients undergoing abdominopelvic operations are known to be at high risk for post-discharge VTE [8]. As a result, current guidelines for abdominopelvic cancer patients call for extended-duration thromboprophylaxis for 4 weeks after surgery, irrespective of the date of discharge from the hospital or ambulatory status $[1,8]$.

Ventral hernia patients are known to be at risk for VTEs $[2,3]$ but there have been no reports examining the risk of VTE after discharge from the hospital, and whether risk factors can be identified to select hernia patients who may benefit from extended-duration thromboprophylaxis. In this study, we used data from the American College of SurgeonsNational Surgical Quality Improvement Program (ACSNSQIP) to investigate the risk of post-discharge VTE. We then identified which patients may be suitable for extendedduration thromboprophylaxis by creating a risk calculator, and validating the calculator on a separate cohort of patients.

\section{Methods}

\section{Patient selection}

ACS-NSQIP prospectively collects procedural information from about 700 hospitals in the United States and Canada and includes patient characteristics, operative variables, complications, and mortality within 30 days of a surgical procedure. We identified all patients who underwent ventral hernia repair reported to ACS-NSQIP from 2011 to 2017 using current procedural terminology (CPT) codes. CPT codes are used in the United States to report medical, surgical, and diagnostic procedures and services to entities such as physicians, health insurance companies, and accreditation organizations. We used following codes: open ventral hernia repair: 49560, 49561, 49565, 49566; laparoscopic ventral hernia repair: 49654, 49655, 49656, 49657; and implantation of mesh: 49568, 15777, 15330, 15331, 15430, 15431. Concurrent procedures were also identified by CPT codes: panniculectomy: 15830; myocutaneous flap: 15734, 15738; concurrent abdominal procedure: 49000, 44005, 47600, 47605, 47563, 44121, 44125, 44130, 44120, 44202, 44602, 44603, 44020, 44227, 44640, 44620, 44346, 58150, 58940; and concurrent bowel procedure: 44121, 44125, 44130, 44120, 44202, 44602, 44603, 44020, 44227, 44640, 44620, 44346. Hernia characteristics were also identified: recurrent hernia: 49565, 49566, 49656, 49657; and incarcerated/strangulated: 49561, 49566, 49655, 49657. The primary outcome was development of a post-discharge VTE, defined as either a deep vein thrombosis or pulmonary embolus that occurred after discharge from the hospital, but within the NSQIP 30-day follow-up period. Deep vein thrombosis was defined in NSQIP as a venous thrombosis, confirmed by a duplex, venogram, CT scan, or other definitive imaging modality, or autopsy; treated with anticoagulation therapy, vena cava filter, clipping of the vena cava, or documentation that treatment was warranted but there was no appropriate treatment option available, or that the patient refused treatment. Pulmonary emboli were defined in NSQIP as new blood clot in a pulmonary artery causing obstruction of the blood supply to the lungs and diagnosed with a V-Q scan interpreted as high probability of pulmonary embolism, trans-esophageal echocardiogram, pulmonary arteriogram, CT angiogram, or any other definitive imaging modality (including autopsy). As this study was performed on a nationally available deidentified dataset, it was exempt from Institutional Review Board Approval.

\section{Model development}

We then created a model that included patient characteristics and procedural details to predict post-discharge VTEs. Patient characteristics included age, Body Mass Index (BMI), sex, race, diabetes mellitus, smoking history, dyspnea, functional status, ventilator dependence, chronic obstructive pulmonary disease, ascites, congestive heart failure, hypertension, acute renal failure, dialysis dependence, disseminated cancer, pre-existing open wound, chronic steroid use, bleeding disorder, preoperative transfusion, preoperative sepsis, total hospital length of stay, days from operation to discharge, discharge destination, and American Society of Anesthesiologists class. Procedural details were operative time, laparoscopic versus open approach, recurrent hernia, incarcerated or strangulated hernia, mesh use, need for myocutaneous flap, panniculectomy, concurrent abdominal procedure, and concurrent bowel procedure. Preoperative laboratory values, such as serum creatinine, albumin, hematocrit, and platelet count, were not included as possible predictors, since there were large amounts of missing data. The NSQIP data set does not contain information as to the thromboprophylaxis strategies (i.e., mechanical versus chemothromboprophylaxis) employed during hospitalization or after discharge. We also assessed reoperation, readmission, and death within 30 days.

\section{Statistical analysis}

Baseline characteristics and outcomes were compared using the Wilcoxon-rank sum test for continuous variables and Pearson $\chi^{2}$ for categorical variables. The risk model was constructed using a primary data set of patients who underwent ventral hernia repair from 2011 to $2016(n=140,301)$ and validated using the cohort of patients undergoing procedures in $2017(n=29,816)$. Predictors with $p$ value $<0.05$ 
in univariate analysis, as well as those known to be associated with VTE were used to construct a multivariable model using forward and backward stepwise regression. The calibration of the model was tested using the Hosmer-Lemeshow goodness-of-fit test, and the discriminatory ability was evaluated using the $c$-statistic. Various cut-points for the calculated risk of post-discharge VTE were assessed using ROC curves, sensitivity, specificity, and Youden's $J$ index (defined as sensitivity + specificity -1 ). An equation to calculate the predicted risk of post-discharge VTE was then created from the model and incorporated into a mobile application. All data analysis was performed using STATA/ MP 16.0 (StataCorp, College Station, TX).

\section{Results}

\section{Risk of post-operative VTE}

VTEs occurred in 878 of 141,065 patients $(0.62 \%)$ within 30 days of surgery. About half of these were deep venous thromboses $(0.33 \%)$ and the other half pulmonary emboli $(0.35 \%)$. When we examined the timing of VTEs relative to hospital discharge, about half (421, or $48 \%$ ) of VTEs occurred after discharge from the hospital. Again, the distribution of these post-discharge VTEs was evenly split between deep vein thrombosis $(245,58 \%)$ and pulmonary embolus $(176,42 \%)$. We then examined the timing of all VTEs relative to the primary hernia operation (Fig. 1). The risk of VTE did not appreciably begin to decrease until 21 days after surgery and was still present at 30 days.

\section{Comparison of patients with post-discharge VTE to those without}

Baseline characteristics of the study cohort, excluding 764 patients who suffered a VTE during their index hospitalization or were still hospitalized at 30 days, are summarized in Table 1. Patients who developed post-discharge VTE were older (61 vs. 57 years, $p<0.001$ ), more obese, (BMI 34 vs. $\left.32 \mathrm{~kg} / \mathrm{m}^{2}, p<0.001\right)$, were more likely to be discharged someplace other than home, and had higher rates of comorbidities: dyspnea, impaired functional status, COPD, CHF, hypertension, cancer, open wounds, bleeding disorders, higher American Society of Anesthesiology class, and more preoperative systemic inflammatory response syndrome. Patients who suffered a post-discharge VTE also had a much longer length of stay in the hospital after their hernia operation (4 vs. 1 days, $p<0.001$ ).

We then compared operative details between cohorts (Table 2). Patients with post-discharge VTE had significantly longer operations ( 2.1 vs. $1.3 \mathrm{~h}, p<0.001)$, and were more likely to be inpatient $(73 \%$ versus $46 \%, p<0.001)$, emergent ( $23 \%$ versus $12 \%, p<0.001)$, and to have a higher wound class $(p<0.001)$. A laparoscopic approach protected patients from post-discharge VTE (15\% versus $21 \%$, $p=0.007)$. Hernia characteristics that increased operative complexity increased the rate of post-discharge VTE: incarceration or strangulation ( $41 \%$ versus $33 \%, p<0.001)$, need for mesh $(52 \%$ versus $46 \%, p=0.011)$, myocutaneous flap (14\% versus $7 \%, p<0.001$ ), panniculectomy (4\% versus $1 \%, p<0.001)$, or concurrent abdominal (22\% versus $12 \%$, $p<0.001)$ or bowel procedure (5\% versus $2 \%, p<0.001)$. Finally, patients who experienced a post-discharge VTE had a higher rate of reoperation $(14 \%$ versus $2 \%, p<0.001)$,
Fig. 1 Histogram of the number of VTEs that occurred on each post-operative day after ventral hernia repair, based on data from the American College of Surgeons-National Surgical Quality Improvement Program (ACS-NSQIP) for the years 2011-2017

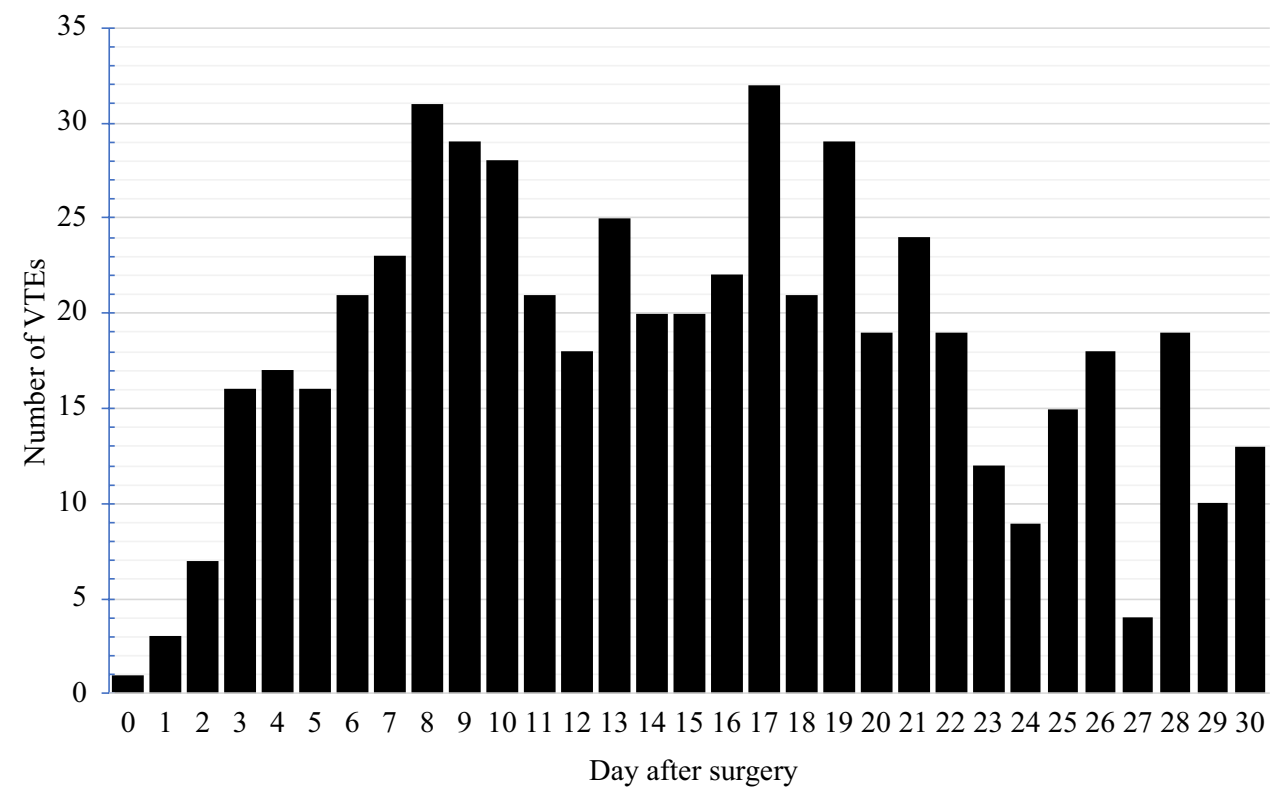


Table 1 Characteristics of patients with ventral hernia repair who developed a VTE after discharge

\begin{tabular}{|c|c|c|c|}
\hline Patient characteristic & No VTE $(n=139,880)$ & $\begin{array}{l}\text { Post-discharge } \\
\text { VTE }(n=421)\end{array}$ & $p$ value \\
\hline Mean age (years) & 57 (47-67) & $61(52-70)$ & $<0.001$ \\
\hline Body mass index $\left(\mathrm{kg} / \mathrm{m}^{2}\right)$ & $31.6(27.4-37.0)$ & $34.1(29.4-40.1)$ & $<0.001$ \\
\hline Female sex $(\%)$ & $80,280(57.4)$ & $222(52.7)$ & 0.15 \\
\hline Discharge destination (\%) & & & $<0.001$ \\
\hline Home & 134,803 (96.6) & $387(92.1)$ & \\
\hline Facility which was home & $732(0.5)$ & $1(0.2)$ & \\
\hline Skilled or unskilled facility & $2498(1.8)$ & $26(6.2)$ & \\
\hline Rehabilitation & $855(0.6)$ & $4(1.0)$ & \\
\hline Separate acute care & $349(0.3)$ & $2(0.5)$ & \\
\hline Hospice & $3(0.0)$ & $0(0.0)$ & \\
\hline Other & $357(0.3)$ & $0(0.0)$ & \\
\hline Diabetes (\%) & & & 0.48 \\
\hline No diabetes & $115,078(82.3)$ & $337(80.1)$ & \\
\hline Not on insulin & $16,543(11.8)$ & $57(13.5)$ & \\
\hline Taking insulin & $8259(5.9)$ & $27(6.4)$ & \\
\hline Smoker $(\%)$ & $26,862(19.2)$ & $70(16.6)$ & 0.18 \\
\hline Dyspnea (\%) & $9.334(6.8)$ & $45(10.7)$ & 0.001 \\
\hline Impaired functional status (\%) & $1860(1.3)$ & $14(3.3)$ & $<0.001$ \\
\hline Ventilator dependent (\%) & $97(0.1)$ & $2(0.5)$ & 0.002 \\
\hline Chronic Obstructive Pulmonary Disease (\%) & $7754(5.5)$ & $37(8.8)$ & 0.004 \\
\hline Ascites (\%) & $711(0.5)$ & $4(1.0)$ & 0.204 \\
\hline Congestive heart failure (\%) & $817(0.6)$ & $4(1.0)$ & 0.325 \\
\hline Hypertension (\%) & $70,268(50.2)$ & $244(58.0)$ & 0.002 \\
\hline Acute renal failure (\%) & $293(0.2)$ & $1(0.2)$ & 0.9 \\
\hline Dialysis dependence (\%) & $1462(1.1)$ & $3(0.7)$ & 0.5 \\
\hline Cancer $(\%)$ & $1007(0.7)$ & $5(1.2)$ & 0.257 \\
\hline Open wound (\%) & $1995(1.4)$ & $16(3.8)$ & $<0.001$ \\
\hline On chronic steroids (\%) & $5993(4.3)$ & $20(4.8)$ & 0.64 \\
\hline Bleeding disorder $(\%)$ & $4446(3.2)$ & $31(7.4)$ & $<0.001$ \\
\hline Preoperative transfusion (\%) & $236(0.2)$ & $0(0.0)$ & 0.4 \\
\hline Systemic Inflammatory Response Syndrome (\%) & $3289(2.4)$ & $21(5.0)$ & $<0.001$ \\
\hline Hospital days after surgery (range) & $1(0-3)$ & $4(1-7)$ & $<0.001$ \\
\hline American Society of Anesthesiology Class & & & $<0.001$ \\
\hline Class I (\%) & $7750(6)$ & $7(2)$ & \\
\hline Class II (\%) & $63,635(46)$ & $137(33)$ & \\
\hline Class III (\%) & $63,329(45)$ & $254(60)$ & \\
\hline Class IV (\%) & $4971(4)$ & $23(6)$ & \\
\hline Class V (\%) & $33(0.02)$ & $0(0)$ & \\
\hline
\end{tabular}

readmission $(73 \%$ versus $6 \%, p<0.001)$, and 30-day mortality $(1.4 \%$ versus $0.4 \%, p<0.001)$.

\section{Construction of predictor model}

Stepwise multivariate regression of predictors resulted in the final multivariable model of eight independent risk factors for prediction of post-discharge VTE (Table 3). Calibration of the model was measured by the Hosmer-Lemeshow goodness-of-fit test, $\chi^{2}=360.19(p=0.711)$, and the discrimination of the model in the training data set was measured by the $c$-statistic $=0.71$. The model was then validated in the 2017 dataset, consisting of 29,816 patients, and the $c$-statistic was 0.64 (95\% CI 0.58-0.69). The equation generated by the multivariable model takes the form of $\hat{p}=\exp$ $\left(b_{0}+b_{1}+b_{2}+\ldots+b_{p}\right) /\left[1+\exp \left(b_{0}+b_{1}+b_{2}+\ldots+b_{p}\right)\right]$, where $\hat{p}$ is the predicted risk of post-discharge VTE, $b_{0}$ is the constant $(-7.12)$, and $b_{1}, b_{2}, \ldots b_{\mathrm{p}}$ are the coefficients 
Table 2 Operative details of patients with ventral hernia repair who developed VTE after discharge from the hospital

Table 3 Multivariable analysis of factors predictive of postdischarge VTE

\begin{tabular}{|c|c|c|c|}
\hline Operative characteristic & No VTE $(n=139,880)$ & $\begin{array}{l}\text { Post-discharge VTE } \\
(n=421)\end{array}$ & $p$ value \\
\hline Mean operative time in hours (range) & $1.3(0.7-2.1)$ & $2.1(1.2-3.3)$ & $<0.001$ \\
\hline Admission required & & & $<0.001$ \\
\hline Outpatient (\%) & $75,206(54)$ & $113(27)$ & \\
\hline Inpatient (\%) & $64,674(46)$ & $308(73)$ & \\
\hline Emergency operation (\%) & $17,339(12)$ & $97(23)$ & $<0.001$ \\
\hline Wound class & & & $<0.001$ \\
\hline Clean $(\%)$ & $122,660(88)$ & $333(79)$ & \\
\hline Clean contaminated $(\%)$ & $12,765(9)$ & $61(15)$ & \\
\hline Contaminated (\%) & $2431(2)$ & $18(4)$ & \\
\hline Dirty/Infected (\%) & $2024(2)$ & $9(2)$ & \\
\hline Laparoscopic approach (\%) & $28,772(21)$ & $64(15)$ & 0.007 \\
\hline Recurrent hernia (\%) & $30,661(22)$ & $107(25)$ & 0.083 \\
\hline Incarcerated or strangulated hernia (\%) & $45,483(33)$ & $173(41)$ & $<0.001$ \\
\hline Mesh used (\%) & $64,163(46)$ & $219(52)$ & 0.011 \\
\hline Myocutaneous flap (\%) & $9028(7)$ & $59(14)$ & $<0.001$ \\
\hline Panniculectomy (\%) & $1649(1)$ & $16(4)$ & $<0.001$ \\
\hline Concurrent abdominal procedure $(\%)$ & $16,567(12)$ & $92(22)$ & $<0.001$ \\
\hline Concurrent bowel procedure (\%) & $2636(2)$ & $19(5)$ & $<0.001$ \\
\hline Reoperation (\%) & $3347(2)$ & $60(14)$ & $<0.001$ \\
\hline Readmission (\%) & $8053(6)$ & $306(73)$ & $<0.001$ \\
\hline Death within 30 days (\%) & $528(0.4)$ & $6(1)$ & $<0.001$ \\
\hline
\end{tabular}

\begin{tabular}{|c|c|c|c|}
\hline Risk Factor & Odds ratio $(95 \% \mathrm{CI})$ & $p$ value & Logit coefficient \\
\hline Age $>60$ years & $1.43(1.17-1.74)$ & $<0.0001$ & 0.36 \\
\hline Male sex & $1.42(1.16-1.73)$ & 0.001 & 0.35 \\
\hline \multicolumn{4}{|c|}{ Body mass index (referenced to BMI $<35 \mathrm{~kg} / \mathrm{m}^{2}$ ) } \\
\hline $35-40 \mathrm{~kg} / \mathrm{m}^{2}$ & $1.36(1.06-1.76)$ & 0.017 & 0.31 \\
\hline $40-45 \mathrm{~kg} / \mathrm{m}^{2}$ & $1.57(1.14-2.15)$ & 0.005 & 0.45 \\
\hline $45-50 \mathrm{~kg} / \mathrm{m}^{2}$ & $1.86(1.25-2.77)$ & 0.002 & 0.62 \\
\hline$>50 \mathrm{~kg} / \mathrm{m}^{2}$ & $1.64(1.12-2.43)$ & 0.013 & 0.50 \\
\hline Operative time $>2 \mathrm{~h}$ & $1.92(1.54-2.38)$ & $<0.0001$ & 0.65 \\
\hline Panniculectomy performed & $2.01(1.20-3.37)$ & 0.008 & 0.7 \\
\hline Postoperative hospitalization $>1$ day & $2.27(1.76-2.91)$ & $<0.0001$ & 0.82 \\
\hline Bleeding disorder ${ }^{\mathrm{a}}$ present & $1.68(1.15-2.44)$ & 0.007 & 0.52 \\
\hline Emergency operation & $1.43(1.11-1.83)$ & 0.005 & 0.35 \\
\hline
\end{tabular}

${ }^{\mathrm{a}} \mathrm{ACS}-\mathrm{NSQIP}$ defines bleeding disorder as any chronic condition that places the patient at risk for excessive bleeding (e.g., vitamin K deficiency, hemophilia, thrombocytopenia, chronic anticoagulation therapy that has not been discontinued prior to surgery, heparin-induced thrombocytopenia, and so on, excluding aspirin therapy and nonsteroidal anti-inflammatory drug use). To calculate the risk of post-discharge VTE, add up the logit function coefficients for each factor present, plus an additional constant of -7.12 . The sum predicts the probability of post-discharge VTE by the logit function, equal to $e^{\mathrm{sum}} /\left(1+e^{\mathrm{sum}}\right)$. This calculation is facilitated by a mobile application for the logit function. This regression equation is the basis for a risk calculator in the form of a free and easy to use mobile application for both iOS (https://apps.apple.com/ us/app/ventral-hernia-vte-risk/id1557362906) and Android devices (https://play.google.com/store/apps/details?id=com. ucsf.crpapp). 


\section{Selection of risk threshold to consider extended-duration thromboprophylaxis}

The predictors listed in Table 3 allow for the calculation of a given patient's risk of post-discharge VTE. For instance, the formula might estimate a given patient's risk of post-discharge VTE to be $0.65 \%$ based on the presence or absence of predictors. The next question becomes, at what risk threshold should surgeons consider extended-duration thromboprophylaxis? To investigate, we evaluated the performance of the risk prediction model in our validation data set using different thresholds (Table 4). For each threshold, we calculated the sensitivity, specificity, Youden's J index, and the AUC. For example, a threshold risk of $>0.3 \%$ would include $36 \%$ of all hernia patients, have a sensitivity of $55 \%$, and have a specificity of $64 \%$ for predicting post-discharge VTE. If a higher threshold of $>0.4 \%$ was chosen, only $25 \%$ of all hernia patients would be included, the sensitivity would decrease to $34 \%$, while the specificity would increase to $75 \%$.

\section{Discussion}

In this study, we found that one VTE occurred for roughly every 160 patients $(0.62 \%)$ who underwent ventral hernia repair. VTEs conferred serious morbidity; they were associated with a readmission in the majority of cases and 2.5fold increased odds of death. Importantly, almost half of the VTEs (48\%) occurred after hospital discharge. Based upon this observation, it seems premature to discontinue thromboprophylaxis in high-risk patients at the time of discharge from the hospital - an arbitrary point in time with respect to VTEs. Indeed, our analysis found that the overall risk of VTE did not begin to diminish until 21 days after the operation and persisted out to 30 days (Fig. 1).

This is not to say that all hernia patients are at high risk for post-discharge VTEs and need extended-duration thromboprophylaxis. We identified eight independent predictors of post-discharge VTE based upon the patient's age, gender, obesity, complexity of hernia repair, and length of stay (Table 3 ). These predictors align with clinical experience. For example, a 75 -year-old man with a BMI $41 \mathrm{~kg} / \mathrm{m}^{2}$, undergoing a 4-h open lysis of adhesions and abdominal wall reconstruction with panniculectomy and requiring a 7-day hospital stay would have a much greater risk of post-discharge VTE (2.2\%) compared to a 45-year-old woman with normal BMI undergoing outpatient ventral hernia repair with operative time $<2 \mathrm{~h}(0.08 \%)$. Notably, technical factors such as longer operative time and need for panniculectomy are also in line with the predictor model for all VTE published by Pannucci [2]. We did not find any association between myocutaneous flap component separation and post-discharge VTE, similar to other studies [9]. Although the final $c$-statistic of the predictive model was 0.64 , meaning that some post-discharge VTEs could not be predicted from the model, the overall accuracy was in line with other predictive models currently in use [2,5]. Additionally, the simplicity of the model adds to its clinical usefulness.

We developed and published a mobile application that surgeons can download into their smartphone (freely downloadable on iOS App Store or Android: "Ventral Hernia VTE Risk Calculator"). The application calculates the postdischarge VTE risk for a given patient. It is our hope that surgeons will use the application to risk-stratify patients and decide whether extended-duration thromboprophylaxis may be of benefit. This approach has proved successful for bariatric surgeons who use the BariatricCalc application, published by the Cleveland Clinic [5].

At what threshold of risk should surgeons consider extended-duration thromboprophylaxis in their ventral hernia patients? This decision should be based upon four factors: (1) the absolute risk of post-discharge VTE without thromboprophylaxis, (2) the number of patients needed to treat to avoid one post-discharge VTE, (3) the risk of adverse events, particularly bleeding, from prescribing extendedduration thromboprophylaxis, and (4) the cost of therapy. Our study addresses the first two factors. To estimate the third factor, the risk of bleeding with thromboprophylaxis, a recent Cochrane analysis estimated the odds of post-operative hemorrhage associated with extended-duration thromboprophylaxis to be 1.1 (95\% confidence interval $0.67-1.81$ ).
Table 4 Sensitivity, specificity, and Youden's $J$ performance for different thresholds of postdischarge VTE risk

\begin{tabular}{lcllll}
\hline Threshold (\%) & \# Patients & Sensitivity (95\% CI) & Specificity (95\% CI) & Youden's J & AUC \\
\hline$\geq 0.1$ & $26,914(90 \%)$ & $96 \%(89-99)$ & $10 \%(9-10)$ & 0.055 & $0.53(0.51-0.55)$ \\
$\geq 0.2$ & $14,274(48 \%)$ & $71 \%(61-80)$ & $52 \%(52-53)$ & 0.23 & $0.62(0.57-0.66)$ \\
$\geq 0.3$ & $10,657(36 \%)$ & $55 \%(44-65)$ & $64 \%(64-65)$ & 0.19 & $0.6(0.54-0.65)$ \\
$\geq 0.4$ & $7536(25 \%)$ & $34 \%(25-45)$ & $75 \%(74-75)$ & 0.09 & $0.55(0.5-0.59)$ \\
$\geq 0.5$ & $5904(20 \%)$ & $32 \%(23-43)$ & $80 \%(80-81)$ & 0.13 & $0.56(0.51-0.61)$ \\
$\geq 0.75$ & $2232(7 \%)$ & $17 \%(10-26)$ & $93 \%(92-93)$ & 0.10 & $0.55(0.51-0.59)$ \\
$\geq 1$ & $982(3 \%)$ & $9 \%(4-16)$ & $97 \%(96-97)$ & 0.05 & $0.53(0.50-0.56)$ \\
$\geq 3$ & $35(0.1 \%)$ & $1 \%(0.0-6)$ & $99 \%(99-100)$ & 0.01 & $0.5(0.49-0.52)$ \\
\hline
\end{tabular}


This very low odds of bleeding was not statistically significant, meaning that extended-duration thromboprophylaxis was not associated with additional bleeding events [10]. As such, the risk of adverse events specifically from extendedduration thromboprophylaxis seems minimal. For the fourth factor, cost of therapy, most centers utilize low-molecular weight heparin in their extended-duration thromboprophylaxis protocols. Unfortunately, these agents are expensive, and often not covered by insurance. As a result, prophylaxis of a large percentage of ventral hernia patients may not be practical $[11,12]$.

To balance factors, we recommend surgeons consider prescribing extended-duration thromboprophylaxis when the calculated risk of post-discharge VTE exceeds $0.3 \%$. This approach would require prophylaxis for only a third of ventral hernia patients (36\%), but this cohort would include over half (55\% sensitivity, $64 \%$ specificity) of the patients who would otherwise develop post-discharge VTEs. At this threshold, the number needed to treat to prevent one postdischarge VTE would be 200. If the cost of therapy were to decrease and no longer be a factor in decision-making, a threshold of $0.2 \%$ risk would include almost half (48\%) of ventral hernia patients to get prophylaxis, but this cohort would include almost three quarters (71\% sensitivity, 52\% specificity) of the patients destined to develop post-discharge VTE. Statistically, the Youden's $J$-statistic also calculates the optimal threshold, based upon the characteristics of the predictor model alone (not taking into account cost, or risk of adverse events). In our study, Youden's $J$-statistic was optimized at a threshold risk of $0.2-0.3 \%$ ( $J$-statistics 0.23 , 0.19 , Table 4$)$, in line with our recommended threshold of $0.3 \%$.

To see if a specific patient's post-discharge VTE risk exceeded $0.3 \%$, surgeons would use the mobile application and enter whether any of the 8 predictors were present. For example, the first patient described above, would have a calculated risk of post-discharge VTE of $2.2 \%$, and would therefore be suitable for extended-duration thromboprophylaxis after discharge. We propose 4 weeks of thromboprophylaxis based upon the observation that risk does not appreciably decrease until post-operative day 21 (Fig. 1). This is in line with recommendations for other high-risk patients undergoing extended-duration thromboprophylaxis $[4-6,8]$.

Our study has several limitations. First, ACS-NSQIP only tracks outcomes within 30 days of surgery. VTE events that occurred later were not captured in the data set, and as such, the true cumulative VTE rate is probably higher than reported here. Second, ACS-NSQIP does not capture all hernia-specific factors that could be predictive of postdischarge VTE, such as hernia dimensions or loss of domain; operative time, need for myocutaneous flap, and panniculectomy serve as surrogates for complexity and size. Third, construction of a predictor model would ideally test all of the variables used in the Caprini scoring system, which is the most popular VTE risk assessment tool today [1, 13-15]. Unfortunately, many of the Caprini risk factors are not contained in ACS-NSQIP, and therefore not available for analysis. Finally, it is impossible to identify which patients received thromboprophylaxis, and what type of prophylaxis they received, both after surgery and after discharge. This information is not contained in ACS-NSQIP. We presume that many patients received prophylaxis while hospitalized, but the number that may have received after discharge is unknown. Hence, our estimate of VTE risk in hospitalized patients is probably less than the true risk would be without any thromboprophylaxis. Nevertheless, despite these limitations, the strength of this study lies in the clinical simplicity of the scoring system and validation using a separate test cohort containing a very large number of hernia patients.

\section{Conclusions}

Almost half of venous thromboembolic events after ventral hernia repair occurred after discharge from the hospital, and the risk of an event did not start to decline until three weeks after surgery. Patients at high risk for post-discharge VTE can be identified based upon patient and procedural characteristics using a simple clinical predictor tool and mobile application. When the risk exceeds $0.3 \%$, extended-duration thromboprophylaxis for 4 weeks can be considered as a strategy to reduce VTE events.

Acknowledgements The authors would like to thank Pamela Derish, Department of Surgery, University of California, San Francisco for contributions to editing the manuscript.

Author contributions SK and JC conceived of the study, designed the analysis, and collected the data. SK performed the data analysis and developed the model with input from JC. DM designed and implemented the mobile applications with input from SK. SK and JC wrote the manuscript with input and approval of all authors.

\section{Declarations}

Conflict of interest The authors have no conflict of interest or disclosures to report.

Ethical approval This study was Institutional Review Board exempt.

Human and animal rights This study is a database study and does not include research directly involving human or animal participation.

Informed consent This study using a nationally available de-identified dataset did not require formal informed consent.

Open Access This article is licensed under a Creative Commons Attribution 4.0 International License, which permits use, sharing, 
adaptation, distribution and reproduction in any medium or format, as long as you give appropriate credit to the original author(s) and the source, provide a link to the Creative Commons licence, and indicate if changes were made. The images or other third party material in this article are included in the article's Creative Commons licence, unless indicated otherwise in a credit line to the material. If material is not included in the article's Creative Commons licence and your intended use is not permitted by statutory regulation or exceeds the permitted use, you will need to obtain permission directly from the copyright holder. To view a copy of this licence, visit http://creativecommons. org/licenses/by/4.0/.

\section{References}

1. Gould MK, Garcia DA, Wren SM et al (2012) Prevention of VTE in nonorthopedic surgical patients. Chest 141(2):e227S-e277S. https://doi.org/10.1378/chest.11-2297

2. Pannucci CJ, Basta MN, Fischer JP, Kovach SJ (2015) Creation and validation of a condition-specific venous thromboembolism risk assessment tool for ventral hernia repair. Surgery 158(5):1304-1313. https://doi.org/10.1016/j.surg.2015.04.001

3. Pannucci CJ, Laird S, Dimick JB, Campbell DA, Henke PK (2014) A validated risk model to predict 90-day VTE events in postsurgical patients. Chest 145(3):567-573. https://doi.org/10.1378/chest. $13-1553$

4. Klaassen Z, Arora K, Goldberg H et al (2018) Extended Venous Thromboembolism Prophylaxis after Radical Cystectomy: A Call for Adherence to Current Guidelines. J Urol 199(4):906-914. https://doi.org/10.1016/j.juro.2017.08.130

5. Aminian A, Andalib A, Khorgami $Z$ et al (2017) Who should get extended thromboprophylaxis after bariatric surgery? A risk assessment tool to guide indications for post-discharge pharmacoprophylaxis. Ann Surg 265(1):143-150. https://doi.org/10.1097/ SLA.0000000000001686

6. Sammour T, Chandra R, Moore JW (2016) Extended venous thromboembolism prophylaxis after colorectal cancer surgery: the current state of the evidence. J Thromb Thrombolysis 42(1):2732. https://doi.org/10.1007/s11239-015-1300-9

7. Moghadamyeghaneh Z, Alizadeh RF, Hanna MH et al (2016) Post-hospital discharge venous thromboembolism in colorectal surgery. World J Surg 40(5):1255-1263. https://doi.org/10.1007/ s00268-015-3361-5
8. Carrier M, Altman AD, Blais N et al (2019) Extended thromboprophylaxis with low-molecular weight heparin (LMWH) following abdominopelvic cancer surgery. Am J Surg 218(3):537-550. https://doi.org/10.1016/j.amjsurg.2018.11.046

9. Kim K, Mella JR, Ibrahim AMS, Koolen PGL, Lin SJ (2015) Is there an association between component separation and venous thromboembolism? Analysis of the NSQIP. Plast Reconstr Surg Glob Open 3(6):e429. https://doi.org/10.1097/GOX.0000000000 000167

10. Felder S, Rasmussen MS, King R et al (2019) Prolonged thromboprophylaxis with low molecular weight heparin for abdominal or pelvic surgery. Cochrane Colorectal Group, ed. Cochrane Database Syst Rev. https://doi.org/10.1002/14651858.CD004318.pub5 ((10))

11. Connell NT, Abel GA, Connors JM (2017) Low-molecular weight heparin versus vitamin $\mathrm{K}$ antagonists for the treatment of cancerassociated thrombosis: a cost-effectiveness analysis. Thromb Res 150:53-58. https://doi.org/10.1016/j.thromres.2016.12.017

12. Robinson R, Wirt TC, Barbosa C et al (2018) Routine use of lowmolecular-weight heparin for deep venous thrombosis prophylaxis after foot and ankle surgery: a cost-effectiveness analysis. J Foot Ankle Surg 57(3):543-551. https://doi.org/10.1053/j.jfas.2017.12. 001

13. Golemi I, Salazar Adum JP, Tafur A, Caprini J (2019) Venous thromboembolism prophylaxis using the Caprini score. Dis Mon 65(8):249-298. https://doi.org/10.1016/j.disamonth.2018.12.005

14. Pannucci CJ, Swistun L, MacDonald JK, Henke PK, Brooke BS (2017) Individualized venous thromboembolism risk stratification using the 2005 Caprini score to identify the benefits and harms of thromboprophylaxis in surgical patients: a meta-analysis. Ann Surg 265(6):1094-1103. https://doi.org/10.1097/SLA.00000 00000002126

15. Sterbling HM, Rosen AK, Hachey KJ et al (2018) Caprini risk model decreases venous thromboembolism rates in thoracic surgery cancer patients. Ann Thorac Surg 105(3):879-885. https:// doi.org/10.1016/j.athoracsur.2017.10.013(15)

Publisher's Note Springer Nature remains neutral with regard to jurisdictional claims in published maps and institutional affiliations. 